This is an electronic reprint of the original article. This reprint may differ from the original in pagination and typographic detail.

Author(s): Chang, Zheng; Gong, Jie; Ristaniemi, Tapani; Niu, Zhisheng

Title: $\quad$ Energy Efficient Resource Allocation and User Scheduling for Collaborative Mobile Clouds with Hybrid Receivers

Year: $\quad 2016$

Version:

Please cite the original version:

Chang, Z., Gong, J., Ristaniemi, T., \& Niu, Z. (2016). Energy Efficient Resource Allocation and User Scheduling for Collaborative Mobile Clouds with Hybrid Receivers. IEEE transactions on vehicular technology, 65(12), 9834-9846. https://doi.org/10.1109/TVT.2016.2525821

All material supplied via JYX is protected by copyright and other intellectual property rights, and duplication or sale of all or part of any of the repository collections is not permitted, except that material may be duplicated by you for your research use or educational purposes in electronic or print form. You must obtain permission for any other use. Electronic or print copies may not be offered, whether for sale or otherwise to anyone who is not an authorised user. 


\title{
Energy Efficient Resource Allocation and User Scheduling for Collaborative Mobile Clouds with Hybrid Receivers
}

\author{
Zheng Chang, Member, IEEE, Jie Gong, Member, IEEE, Tapani Ristaniemi, Senior Member, IEEE, \\ Zhisheng Niu, Fellow, IEEE
}

\begin{abstract}
In this paper, we study the resource allocation and user scheduling algorithm for minimizing the energy cost of data transmission in the context of OFDMA collaborative mobile cloud (CMC) with simultaneous wireless information and power transfer (SWIPT) receivers. The CMC, which consists of several collaborating mobile terminals (MTs) offers one potential solution for downlink content distribution and for the energy consumption reduction. Previous work on the design of CMC system mainly focused on the cloud formulation or EE investigation, while how to allocate the radio resource and schedule user transmission lacks attention. With the objective to minimize the system energy consumption, an optimization problem which jointly considers subchannel assignment, power allocation and user scheduling has been presented. We propose different algorithms to address the formulated problem based on the convex optimization technique. Simulation results demonstrate that the proposed user scheduling and resource allocation algorithms can achieve significant EE performance.
\end{abstract}

Index Terms-content distribution; green communications; resource allocation; subchannel allocation; power allocation; user scheduling; collaborative mobile clouds; user cooperation.

\section{INTRODUCTION}

\section{A. Background}

The advanced wireless communications systems bring to the end-users new high speed services and features that have never been expected in the past. Nevertheless, the dramatic growth in Internet traffics and the growing pervasive use of mobile technologies continuously and significantly increase $\mathrm{CO}_{2}$ emissions in the future unless new energy efficient mechanisms can be investigated. Nowadays energy crisis and global warming problems are urging much research work on

Copyright (c) 2015 IEEE. Personal use of this material is permitted. However, permission to use this material for any other purposes must be obtained from the IEEE by sending a request to pubs-permissions@ieee.org

Z. Chang and T. Ristaniemi are with University of Jyvaskyla, Department of Mathematical Information Technology, P.O.Box 35, FIN-40014 Jyvaskyla, Finland. J. Gong is with School of Data and Computer Science, Sun Yatsen University, Guangzhou, Guangdong Province, China, and Z. Niu is with Tsinghua National Laboratory for Information Science and Technology, Department of Electronic Engineering, Tsinghua University, Beijing 100084, P. R. China. e-mail: zheng.chang@jyu.fi, gongj26@mail.sysu.edu.cn, tapani.ristaniemi@jyu.fi, niuzhs@tsinghua.edu.cn. The paper has been presented in part at IEEE INFOCOM 2014 Workshop on Green Cognitive Communications and Computer Networking, Toronto, Canada, Apr. 2014.

This work is sponsored in part by the Academy of Finland (Decision number 284748, 288473) and by the National Basic Research Program of China (973 Program: No. 2012CB316001), the National Science Foundation of China (NSFC) under grant No. 61461136004.

Manuscript received; revised. energy efficiency study. In the ICT industry, which is becoming one of the major consumer of global energy consumption, researchers have investigated various approaches for energy saving. On the other hand, the conventional wireless networks are energy-constrained, in the sense that the network elements, such as mobile phones, are equipped with batteries. The battery capacity is improved at relatively slow speed during the last decades, which creates the bottleneck in prolonging the lifetime of the networks. Meanwhile, the rise of online services significantly increases the demand for high data rate transmissions, hence straining the current network as well as drawing battery of mobile devices much faster than before. All these issues have resulted in an increased interest in energy efficient research to reduce the energy requirements for wireless communications, and can thus contribute not only to goals for sustainable development, but also to the profitability of the telecommunication industry and user experience improvement. So called green communications, including the design of energy-efficient communication infrastructures, protocols, devices, and services, become inevitable trends for the evolution of the future networks. In this work, we are also aiming to propose novel approaches to reduce the network energy cost, especially from the terminal aspect.

\section{B. Related Work}

Conventional studies on energy-constrained networks usually focused on designing the energy saving scheme proposals. Recently, energy harvesting $(\mathrm{EH})$ technique received considerable attention due to its capability of realizing selfmaintenance in wireless networks. Meanwhile, researches on $\mathrm{EH}$ have mainly concentrated on the transmitter side [1]. That is, for a cellular network, the recent work mainly focused on the development of Base Station (BS) to utilize the harvested energy rather than the mobile nodes, e.g., in [2][3]. Although, there are many EH resources, such as solar, wind and tide, they are usually either location- or weather-dependent. Thus, for the indoor users who can not access solar or wind, EH becomes luxury or even impossible. These constraints motivate the wireless power transfer technology for the terminals, which enables the radio receiver to capture the radio frequency (RF) radiation and convert into a direct current voltage [4].

As RF signal can carry both information and energy simultaneously, the induced simultaneous wireless information and power transfer (SWIPT) has gained much attention [5] [6]. 
Through SWIPT, the receiver not only can receive data from the transmitter, but also can recycle the transmit power to prolong the battery lifetime. Nowadays, electromagnetic wave is almost everywhere all around people's life, so enabling SWIPT is full of possibility in the future wireless networks. In [4] and [5], the fundamental trade-offs between wireless information and power transfer were studied with the assumption that the receiver can simultaneously receive information and harvest energy from the RF signal. In [7] and [8], the authors proposed a receiver architecture which can split the received power into two power streams to facility the SWIPT. The authors of [7] and [8] also investigated the rate-energy regions for a SWIPT receiver in a two-user P2P scenario. The authors of [9] focused on the optimization problem of power control and scheduling for SWIPT receiver. The optimal information decoding (ID) and EH mode switching was then derived. The work in [10] studied the outage probability of a cooperative EH relay network with multiple transmission pairs. In [11] and [12], different subcarrier and power allocation algorithms were proposed for the multiuser Orthogonal Frequency-Division Multiplexing (OFDM) systems with SWIPT. Non-convex optimization problem was formulated with the objective to maximize the energy efficiency performance in term of bits / Joule. For a large scale Multi-Input Multi-Output (MIMO) system with SWIPT receivers, the authors of [13] presented an optimization scheme to maximize the energy efficiency of the system while satisfying the delay constraints by jointly allocating the transmission time duration and transmit power.

Meanwhile, the multimedia services, such as news download (e.g., breaking news), multimedia multicasting (e.g., live sport events or videos), or content distribution (e.g., device configuration files/pictures), are facing increasingly popularity in the daily life. Hence, how to efficiently provide those services for various users also attracts many interests. One of the potential solutions for content distribution in the mobile networks is to design a cooperative content distribution architecture named Collaborative Mobile Cloud (CMC) where the users can share some content and information cooperatively through Device-to-Device (D2D) or Machineto-Machine (M2M) communications [14]. In addition, CMC is foreseeable to reduce the energy consumption of mobile terminals (MTs) as well [16]. Different from the concepts of cloud computing and cloud radio access network (CRAN) where a centralized entity with rich computational resources executes the computing or data processing tasks instead of MT [17][18], CMC is formed by a number of MTs who can process data in a distritbuted and cooperative manner. In the $\mathrm{CMC}$, the MTs can actively use two wireless interfaces: one to communicate with the BS over a Long-Range (LR) wireless technology (such as LTE) and the other to cooperate with other MTs over a Short-Range (SR) communication link (such as WLAN/ac hoc) [15]. In the traditional service, each MT has to download the whole content on its own, which leads to significant energy consumption, especially if the LR data rate is low. In the CMC system, several MTs can form a coalition and each MT receives parts of required information data from BS. Then they exchange the received data with others [16]. In such a case, each MT only needs to download parts of the data and consequently, the receiving time can be significantly reduced. Although information exchange over SR introduces new transmission overhead, the energy consumption can still be significantly decreased as the SR is generally more efficient in term of data rate [19].

As SWIPT enables the traditional information receiver to harvest energy from transmission signal, a CMC formed by a group of SWIPT receivers is expected to improve energy saving performance. Moreover, other than the energy efficiency benefits in technology domain, enabling the wireless power transfer is able to bring novel insights in the cloud formulation. It can be found that the user who is selected for data transmission may consume more energy when transmitting data. Although in the long term it can obtain energy savings, the selfish nature of the user may prevent user to be selected. Therefore, using wireless power transfer is, in deed, one catalyst for the users to join the cloud as the additional energy can be obtained from the BS. Previous work on the design of CMC mainly focused on the cloud formulation or energy efficiency investigations, how to allocate the limited radio resources such as subchannels or transmit power lacks concerns. However, as the future wireless network is OFDMAbased, efficient and practical radio resource allocation scheme is critical. Intuitively, there are two ways for delivering data between BS and CMC. One is that BS can transmit different packets to different MTs in a parallel way. In this case, more channel bandwidths are needed as BS is transmitting to different MTs with different data simultaneously. The other one is that BS can transmit to the MTs in a sequence. For each data segment, one MT is selected as the receiver for BS and transmitter for other MTs. As such, channel bandwidth can be effectively utilized. In this work, we focus on the latter case. On the way towards resource allocation for CMC with SWIPT receivers, there are some obstacles which need more effort:

- How to schedule the proper MT to receive from BS and transmit to other MTs is essential. As CMC is expected as an energy-efficient content distribution system, the MTs being selected should be able to minimize the overall system energy cost;

- How to properly assign the subchannels for the transmission between $\mathrm{BS}$ and CMC and the transmission inside CMC should also be concentrated. Moreover, power allocation schemes for both BS and MTs need to be investigated so that the transmit power consumption can be minimized;

- The ID and EH functionalities of receivers make the problem even more complicated. At each scheduling time, which MT should be selected so that the harvested energy can be maximized while the receive energy consumption can be minimized? In addition, since eventually better channel condition and higher transmit power of BS can bring more energy for harvesting, which set of subchannnels and how much transmit power should be used so that the sum of the consumed energy and harvested energy can be minimized call for contiguous consideration. 


\section{Contributions}

Some of the previous works on designing the energy efficient content distribution platform usually concentrated on the platform formulation [19][20][23]. The authors in this line of work studied the group or coalition formulation problem where several groups can be formulated, and each group can select one user as the head for receiving data from BS [19] [20]. The authors of [20] also proposed scheduling algorithm for the LR to select proper resource to carry out the downlink transmission. It is worth noticing that the considered system model can be applied to the vehicular networks as well for data download, storage and sharing as well. In the vehicular networks, the proposed road-side units (RSUs) are deployed to assist the data download and sharing [21] and [22]. Different from other previous work, we address the user scheduling and resource allocation problem for both LR and SR other than cloud formulation for the considered system. Moreover, this work is also the first in this area attempting to provide resource allocation solutions to a wireless power transfer inspired mobile cloud platforms. In order to utilize the channel bandwidth, during the BS data delivery process, one dedicated receiver, denoted as information decoding MT (IMT), will receive the assigned data and the other MTs, denoted as energy harvesting MTs (EMTs), will harvest energy from the signal. As such, the transmission of the system is in a sequence and no extra bandwidth other than the conventional multicasting is required. After receiving from BS, IMT will share the data to other EMTs of the previous stage. Solving the formulated problem is challenging due to the aforementioned obstacles. To address the formulated the problem, different nonlinear programming methods are applied. Initial results of this work have been published in [24], while in this work, we tackle challenges on this subject more in depth and provide fundamental analysis. The main contribution over previous existing works is three-fold:

- We first model the energy consumption of the overall transmission process when considering baseband circuit energy consumption, RF transmit and receive energy consumption and harvested energy;

- Then we focus on the algorithm design aspect and propose a joint power allocation, subchannel allocation and user scheduling scheme with the objective of optimizing energy consumption performance of CMC with SWIPT;

- By using nonlinear fractional programming optimization techniques and iterative algorithm design, we address the formulated user scheduling and resource allocation problem. Simulation results are presented to illustrate the energy saving gain of the proposed scheme.

The rest of this paper is organized as follows. Section II describes the collaborative mobile cloud system model. In Section III, energy consumption models of ID and $\mathrm{EH}$ receivers, as well as CMC are presented. In Section IV, we formulate the optimization problem and introduce resource allocation and user scheduling solution. We demonstrate the benefits of our proposed algorithm in Section $\mathrm{V}$ through simulation study and finally conclude this work in Section VI

\section{SySTEM MODEL}

In our considered OFDMA system, it is assumed that $K$ MTs locating geographically close are interested in downloading the same content from a BS using one LR wireless technology (e.g., UMTS/HSPA, WiMAX, or LTE). In the CMC system, a MT firstly receives parts of the data and then shares with each other through SR transmission via D2D/M2M connections [16]. As D2D/M2M connections are able to offer better data rate than LR, then the receive time durations of other MTs on SR link can be reduced when receiving a certain amount of data. The MTs are able to decode information and harvest energy from the received radio signals. All transceivers are equipped with a single antenna. Moreover, We assume the channel follows quasi-static block fading and the channel state information (CSI) can be accurately obtained by the feedback from the receivers. Our proposed scheme can be extended to the case that the CSI can not be perfectly obtained by the BS. While the focus will be in turn to examine the CSI imperfection effect. It can be also noticed that the feedback transmission from the MTs to the BS may induce additional energy consumption. We consider the feedback can be realized through the standard feedback mechanisms and channels. Correspondingly, the energy consumption on feedback channels is assumed by to constant and does not bring additional influence to the presented system and schemes. Therefore, to concentrate on the radio resource allocation scheme in the downlink, in this work, we will not take into consideration the energy consumption on the feedbacks, channel state information delivery or channel training frame.

The transmission process of CMC is depicted in Fig. 1 comparing with the conventional transmission, e.g., unicasting/multicasting. In Fig. 1, the CMC consists of three MTs. To simply present the concept of the $\mathrm{CMC}$, we divide the overall data stream into 9 segments. In a conventional setup, the communication interface of a MT (e.g. stand-alone MT) has to remain active for the whole reception duration. This results in high energy consumption due to the required $\mathrm{RF}$ and baseband processing during data reception. In the CMC, BS can distribute various and exclusive segments of data (3 segments in the figure) to different MTs, and MTs can then exchange/share the received parts with other coalition members by utilizing the SR transmission among MTs. Thus, the reception duration can be seriously reduced compared to the conventional transmission given that SR has better a data rate than LR because of, e.g., its better channel condition. The time sequence of the transmission process is shown in Fig. 2 where how the transmission duration can be reduced can be found. However, in order to share the received data, additional communication overheads are induced, such as transmit power of SR transmitter, transmit/receive durations. Consequently, the inherent energy efficiency performance calls for a careful algorithm design [25].

In order to focus on the resource allocation algorithm design and isolate it from the specific hardware implementation details, we do not assume a particular type of $\mathrm{EH}$ receiver. In this work, we focus on the receivers which consist of an energy harvesting unit and a conventional signal processing 


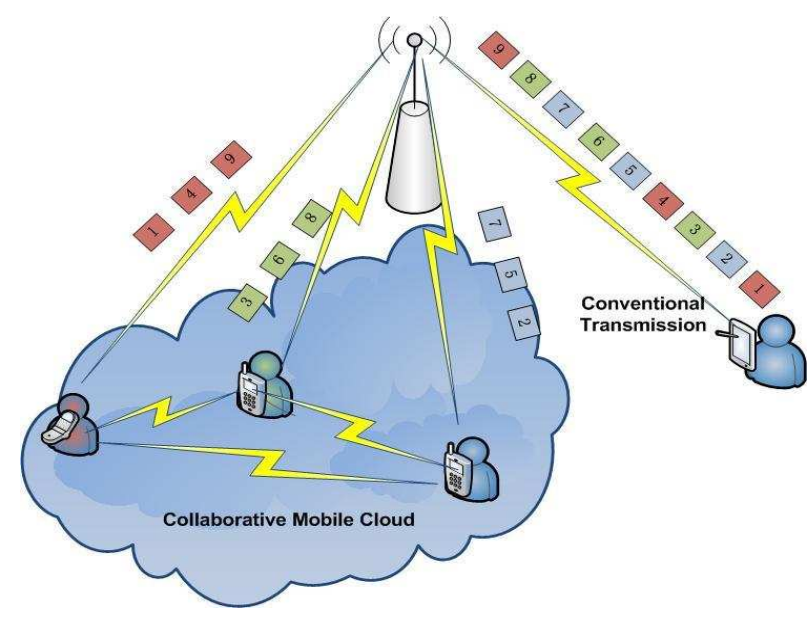

Figure 1. Collaborative mobile cloud

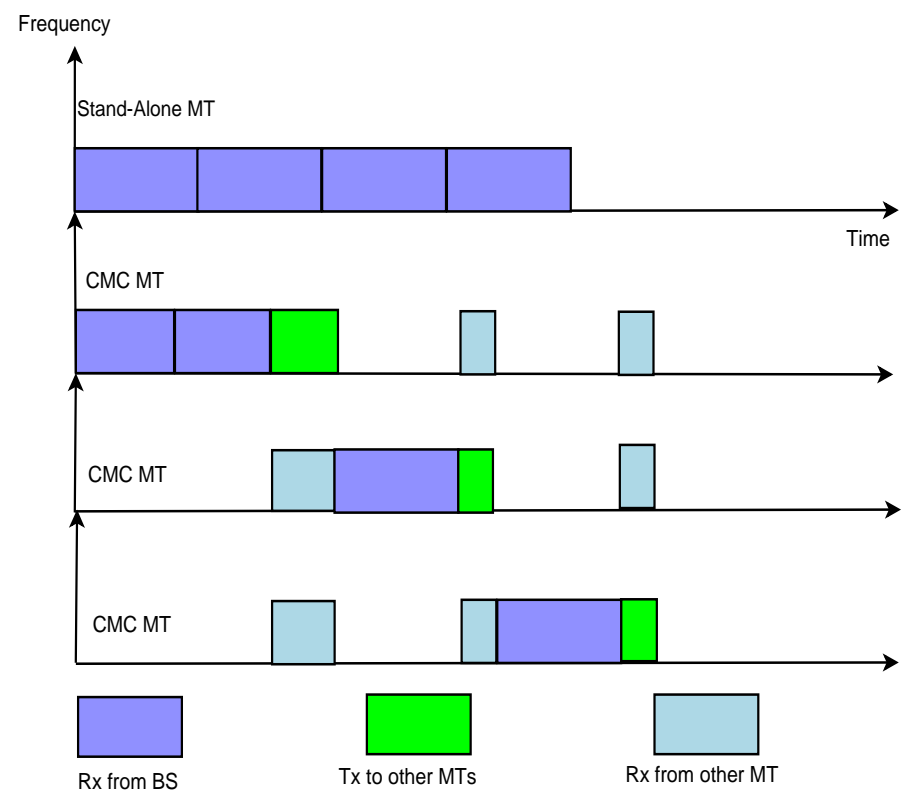

Figure 2. Transmission procedure

core unit for energy harvesting and information decoding, respectively. In addition, for the conventional signal processing, we separate receiver architecture into two parts, which are RF unit and baseband unit. In the following section, the energy consumption model of the considered system is presented.

\section{Energy Consumption Model}

\section{A. Channel Model}

We consider an OFDMA system where a BS services $\mathrm{K}$ mobile receivers. In particular, each receiver is able to decode information and harvest energy from the received radio signals. At first, the BS transmits data to the MT $k$. Thus, let $x$ be the transmitted data from $\mathrm{BS}$, the received data on subchannel $i$ at MT $k$ can be modeled as

$$
y_{1}=\sqrt{P_{s, k, i}^{L_{t x}} L_{s, k} H_{s, k, i}} x+z_{s, k, i},
$$

where $P_{s, k, i}^{L_{t x}}$ and $H_{s, k, i}$ are the transmit power and channel fading gain on subchannel $i$ from BS to MT $k$, respectively. $L_{s, k}$ represents the path loss from BS to MT $k . z_{s, k, i}$ is the Gaussian additive noise with zero mean and variance $\sigma_{z}^{2}$.

After receiving from BS, the MT $k$ is able to deliver the data to other MTs. For the inter-devices multicasting among MTs inside CMC, the received signal at subchannel $j$ can be modeled as

$$
y_{2}=\sqrt{P_{k, j}^{S_{t x}} L_{k} H_{k, j}} x+z_{k, j},
$$

where $P_{k, j}^{S_{t x}}$ is the multicast transmit power of MT $k$ on subchannel $j, L_{k}$ and $H_{k, j}$ are the path loss and channel gain from $k$ to the MT with worst channel condition, respectively.

\section{B. Energy Harvesting Receiver}

In practice, the model of an $\mathrm{EH}$ receiver depends on its specific implementation. For example, electromagnetic induction and electromagnetic radiation are able to transfer wireless power, and the receiver is able to recycle the wireless power from radio signal [5]. Nevertheless, the associated hardware circuit in the receivers and the corresponding EH efficiency can be different. Besides, the signal used for decoding the modulated information cannot be used for harvesting energy due to hardware limitations [7]. In this work, we do not go into the details of any particular type of EH receiver and the general energy harvesting receiver model is used, e.g., the one in [8], [10] and [11].

We denote $P_{s, n, i}^{H}$ as the harvested power on subchannel $i$ by EMT $n$. Then we have [8]

$$
P_{s, n, i}^{H}=\vartheta_{n} P_{s, n, i}^{L_{t x}} L_{s, n} H_{s, n, i},
$$

where we assume the conversion efficiency $0<\vartheta_{n} \leq 1$.

\section{Information Decoding Receiver}

For the data rate on LR $R_{s, k, i}^{L}$, the maximum achievable data rate in $b p s / H z$ from $\mathrm{BS}$ to MT $k$ on subchannel $i$ is given as

$$
R_{s, k, i}^{L}=\log _{2}\left(1+\frac{P_{s, k, i}^{L_{t x}} L_{s, k} H_{s, k, i}}{\sigma_{z}^{2}}\right) .
$$

In the CMC, the IMT $k$ needs to multicast its received data to other CMC members, so the data rate on the subchannel $j$ of SR link can be expressed as

$$
R_{k, j}^{S}=\log _{2}\left(1+\frac{P_{k, j}^{S_{t x}} L_{k} H_{k, j}}{\sigma_{z}^{2}}\right),
$$

where we also assume that the noises on LR and SR are of same kind for simplicity. 


\section{Tx and Rx Energy Consumption of CMC}

As we know, the energy consumption can be modeled as a linear function containing the power consumption and the time duration. Therefore, the energy consumption for receiving data size $S_{T}$ from BS can be expressed as

$E_{s, k, i}^{L_{r x}}=\left(P_{s, k, i}^{L_{r x}}+P_{E}\right) T_{s, k, i}^{L_{r x}}=\frac{\left(P_{s, k, i}^{L_{r x}}+P_{E}\right) S_{T}}{R_{s, k, i}^{L}}=\frac{\left(P_{r x}+P_{E}\right)}{R_{s, k, i}^{L}}$

where $P_{s, k, i}^{L_{r x}}$ is the RF power consumption of $k$ for receiving from BS on subchannel $i$ and $P_{E}$ is the electronic circuit power consumption of baseband associated with transmission bandwidth. The nonlinear effect of power amplifier is ignored. In this work, the energy consumption refers to the one when receiving and sending data on certain subchannel, so the baseband power consumption is considered together with RF Tx/Rx power consumption. $T_{s, k, i}^{L_{r x}}=\frac{S_{T}}{R_{s, k, i}^{L}}$ is the required time for receiving data $S_{T}$ on LR subchannel $i$. Further we can assume the receive RF power consumption are the same for both LR and SR links, and equals to $P_{r x}$. After receiving from the $\mathrm{BS}, \mathrm{MT} k$ is going to transmit its received data to other required MTs. There are two conventional ways to deliver data inside CMC, which are unicasting and multicasting. We have discussed the energy efficiency of using both two schemes in [25] and the multicasting shown the superior energy efficiency performance over unicasting. So in this work, we only invoke multicasting as the transmission strategy inside CMC.

When multicasting is used, an IMT only needs to broadcast its data to other MTs in CMC once with the data rate that can reach the MT with the worst channel condition. Thus the transmit energy consumption of IMT is given as

$$
E_{k, j}^{S_{t x}}=\left(P_{k, j}^{S_{t x}}+P_{E}\right) T_{k, j}^{S_{t x}}=\frac{\left(P_{k, j}^{S_{t x}}+P_{E}\right) S_{T}}{R_{k, j}^{S}} .
$$

Therefore, the total energy consumption of CMC when using MT $k$ as the IMT can be expressed as follows:

$$
E_{k, i, j}=E_{s, k, i}^{L_{r x}}+E_{k, j}^{S_{t x}}+\sum_{n, n \neq k}^{K} E_{n, j}^{S_{r x}} .
$$

$E_{k, i, j}$ is the energy consumption of IMT $k$ when assigning subchannel $i$ for receiving from BS and subchannel $j$ for broadcasting its received data. $E_{n, j}^{S_{r x}}$ is the energy consumption of each EMT when receiving from IMT on subchannel $j$, and it can be expressed as

$$
E_{n, j}^{S_{r x}}=\left(P_{n, j}^{S_{r x}}+P_{E}\right) T_{k, j}^{S_{r x}}=\frac{\left(P_{n, j}^{S_{r x}}+P_{E}\right) S_{T}}{R_{k, j}^{S}}=\frac{\left(P_{r x}+P_{E}\right) S_{T}}{R_{k, j}^{S}}
$$

\section{E. Energy Consumption of Base Station}

The energy consumption of the BS is given as

$$
E_{s}^{L_{t x}}=\left(P_{s, k, i}^{L_{t x}}+P_{B}\right) T_{s, k, i}^{L_{r x}}=\frac{\left(P_{s, k, i}^{L_{t x}}+P_{B}\right) S_{T}}{R_{s, k, i}^{L}},
$$

where $P_{B}$ is the BS baseband operating power consumption.

\section{Resource Allocation And User Scheduling}

\section{A. Problem Formulation}

In the previous section, we presented the energy consumption model of the CMC with hybrid ID-EH receivers in each scheduling time slot. In this section, the considered problem will be formulated as a joint optimization of subchannel Sallocation, power allocation and user scheduling with the objective to minimize the energy consumption during each transmission time of sending data $S_{T}$.

In order to minimize the energy consumption of a $\mathrm{CMC}$, at first one MT inside CMC will be selected as the IMT and other MTs will be considered as EH receivers. Then the BS transmits the data to the IMT on the selected subchannel $i$ with data rate $R_{s, k, i}^{L}$. After that, the IMT will act as a relay and forwards the received data to other MTs on the selected subchannel $j$ with multicast data rate $R_{k, j}^{S}$. In this work, we tackle the problem when one subchannel is used for LR link and one is used for SR link. However, the work can be applied to multiple-subchannel problems as well.

To this end, taken harvested energy into consideration, for each data segment transmission, we can formulate the optimization objective as

$$
\begin{aligned}
\mathcal{E}(\boldsymbol{\rho}, \boldsymbol{\omega}, \mathbf{P}) & =\sum_{k=1}^{K} \sum_{i=1}^{N} \rho_{k} \omega_{s, k, i} E_{s}^{L_{t x}}+\sum_{k=1}^{K} \sum_{i=1}^{N} \sum_{j=1}^{N} \omega_{s, k, i} \omega_{k, j} \rho_{k} E_{k, i, j} \\
& -\sum_{i=1}^{N} \sum_{k=1}^{K} \sum_{n, n \neq k} \omega_{s, k, i} \rho_{k} Q_{s, n, i},
\end{aligned}
$$

where $N$ is the number of available subchannels, and $K$ is the total number of MTs inside CMC. $\mathbf{P}$ is the power allocation policy. $\boldsymbol{\rho}=\left\{\rho_{k}\right\}, \forall k$ and $\boldsymbol{\omega}=\left\{\omega_{s, k, i}, \omega_{k, j}\right\}, \forall k, i, j$ are the user selection and subchannel allocation indicators. In this work, there is no constraint on whether $i$ and $j$ can be the same since no interference will be occurred. $Q_{s, n, i}$ is the harvest energy and obversely $Q_{s, n, i}=P_{s, n, i}^{H} S_{T} / R_{s, k, i}^{L}$. Note that mathematically $\mathcal{E}(\boldsymbol{\rho}, \boldsymbol{\omega}, \mathbf{P})$ can take the negative value. However, the case that $\mathcal{E}(\boldsymbol{\rho}, \boldsymbol{\omega}, \mathbf{P})$ is positive always holds for practical system. The defined binary variable $\rho_{k}$ is the indicator whether MT $k$ is selected as IMT, that is,

$$
\rho_{k}= \begin{cases}1, & \text { if } k \text { is chosen as IMT for receiving from BS, } \\ 0, & \text { otherwise. }\end{cases}
$$

In addition, we also define $\omega$ is the indicator whether certain subchannel is assigned to MT $k$, e.g.,

$$
\omega_{s, k, i}= \begin{cases}1, & \text { if subchannel } i \text { is used by } k \text { for downlink, } \\ 0, & \text { otherwise. }\end{cases}
$$

and

$\omega_{k, j}= \begin{cases}1, & \text { if subchannel } j \text { is assigned to } k \text { to deliver data } \\ 0, & \text { otherwise. }\end{cases}$ 
Therefore, the user selection and resource allocation optimization problem can be formulated as

$$
\min _{\boldsymbol{\rho}, \boldsymbol{\omega}, \mathbf{P}} \mathcal{E}(\boldsymbol{\rho}, \boldsymbol{\omega}, \mathbf{P})
$$

s.t.

$$
\begin{aligned}
C 1: & \sum_{k=1}^{K} \rho_{k}=1, \\
C 2: & \sum_{k=1}^{K} \omega_{s, k, i}=1, \sum_{k=1}^{K} \omega_{k, j}=1, \\
C 3: & R_{s, k, i}^{L} \geq R_{L, \min }, \\
C 4: & R_{k, j}^{S} \geq R_{S, \min }, \\
C 5: & \sum_{k=1}^{K} \sum_{i=1}^{N} \rho_{k} \omega_{s, k, i} P_{s, k, i}^{L_{t x} \leq P_{s, \max },} \\
C 6: & \sum_{k=1}^{K} \sum_{j=1}^{N} \rho_{k} \omega_{k, j} P_{k, j}^{S_{t x}} \leq P_{k, \max },
\end{aligned}
$$

Here, the optimization problem (15) is formulated with several constraints. The first constraint $\mathrm{C} 1$ is to ensure only one user is selected for receiving data from BS in a scheduled slot. C2 ensures the subchannels allocated to MT $k$ are unique. $R_{L, \text { min }}$ in $\mathrm{C} 3$ and $R_{S, \text { min }}$ in $\mathrm{C} 4$ are the required data rates for LR and SR, respectively. C5 and C6 ensure that the power allocation of BS and IMT should not be higher than the maximum allowed transmit power.

It is worth noticing that (15) with (16) is combinatorial in nature with a non-convex structure. In general, there is no standard approach for solving such a non-convex optimization problems and such integer programming problem is recognized as NP-hard. In the extreme case, an exhaustive search or branch-and-bound method is needed to obtain the global optimal solution which requires high computational complexity even for small $K$ and $N$. In order to make the problem tractable, we transform the objective function and approximate the transformed objective function in order to simplify the problem.

\section{B. Power and Subchannel Allocation Scheme}

Theorem 1. The objective function (15) is quasi-convex function w.r.t.e to the power allocation variables $P_{s, k, i}^{L_{t x}}$ and $P_{k, j}^{S_{t x}}$, respectively.

The proof of Theorem 1 can be found in Appendix A. Thus, as a result, the unique global optimal solutions for $P_{s, k, i}^{L_{t x}}$ and $P_{k, j}^{S_{t x}}$ exists and the optimal point can be obtained by using the bisection method [26]. We can also apply the nonlinear fractional programming method to solve the formulated problem [27] of power allocation and subchannel allocation in the followings.

1) Problem Transformation: First given that the user scheduling is done, i.e. $\rho_{k}=1$, we can reform the objective function $\mathcal{E}(\boldsymbol{\rho}, \boldsymbol{\omega}, \mathbf{P})$ as a function of $\{\boldsymbol{\omega}, \mathbf{P}\}$. Substituting (3), (8) and (10) into (11), we can arrive (17) where $P_{c}=$
$P_{B}+P_{r x}+P_{E}$. One may notice that obtaining power allocation policy involves solving $\mathcal{E}(\boldsymbol{\omega}, \mathbf{P})$, which can be expressed as

$$
\mathcal{E}(\boldsymbol{\omega}, \mathbf{P})=\mathcal{E}_{L R}\left(\omega_{s, k, i}, P_{s, k, i}^{L_{t x}}\right)+\mathcal{E}_{S R}\left(\omega_{k, j}, P_{k, j}^{S_{t x}}\right),
$$

where $\mathcal{E}_{L R}\left(\omega_{s, k, i}, P_{s, k, i}^{L_{t x}}\right)=\frac{U_{1}\left(\omega_{s, k, i}, P_{s, k, i}^{L_{t x}}\right)}{R_{1}\left(\omega_{s, k, i}, P_{s, k, i}^{L_{t x}}\right)} \quad$ and $\mathcal{E}_{S R}\left(\omega_{k, j}, P_{k, j}^{S_{t x}}\right)=\frac{U_{2}\left(\omega_{k, j}, P_{k, j}^{S_{t x}}\right)}{R_{2}\left(\omega_{k, j}, P_{k, j}^{S_{t x}}\right)}$. From (18), one can observe that the power allocation schemes for BS and scheduled MT are separated. In other word, we can obtain optimal power allocation by addressing $\mathcal{E}_{L R}\left(\omega_{s, k, i}, P_{s, k, i}^{L_{t x}}\right)$ and $\mathcal{E}_{S R}\left(\omega_{k, j}, P_{k, j}^{S_{t x}}\right)$ individually when user scheduling is done. We can see that both $\mathcal{E}_{L R}\left(\omega_{s, k, i}, P_{s, k, i}^{L_{t x}}\right)$ and $\mathcal{E}_{S R}\left(\omega_{k, j}, P_{k, j}^{S_{t x}}\right)$ are quasi-convex functions w.r.t. power allocation variables. For the sake of presentation simplicity, we introduce a method for solving $\mathcal{E}_{L R}\left(\omega_{s, k, i}, P_{s, k, i}^{L_{t x}}\right)$ which is derived from nonlinear fractional programming [27].

The global optimal solution $q_{L R}^{*}$ can be expressed as

$$
q_{L R}^{*}=\mathcal{E}_{L R}\left(\omega_{s, k, i}^{*}, P_{s, k, i}^{L *}\right)=\min _{\omega_{s, k, i}, P_{s, k, i}^{L_{t x}}} \frac{U_{1}\left(\omega_{s, k, i}, P_{s, k, i}^{L_{t x}}\right)}{R_{1}\left(\omega_{s, k, i}, P_{s, k, i}^{L_{t x}}\right)} .
$$

Theorem 2. The optimal solution $q_{L R}^{*}$ can be obtained iff

$$
\min _{\omega_{s, k, i}, P_{s, k, i}^{L_{t x}}} U_{1}\left(\omega_{s, k, i}, P_{s, k, i}^{L_{t x}}\right)-q_{L R}^{*} R_{1}\left(\omega_{s, k, i}, P_{s, k, i}^{L_{t x}}\right)=0 .
$$

Theorem 2 gives a necessary and sufficient condition w.r.t. optimal power allocation. The proof can be found in Appendix B. Particularly, for the considered optimization problem with an objective function in fractional form, there exists an equivalent optimization problem with an objective function in subtractive form, i.e., $U_{1}\left(\omega_{s, k, i}, P_{s, k, i}^{L_{t x}}\right)-q_{L R}^{*} R_{1}\left(\omega_{s, k, i}, P_{s, k, i}^{L_{t x}}\right)$, and both formulations result in the same power allocations. To achieve the optimal $q_{L R}^{*}$, the iterative algorithm with guaranteed convergence in [27] can be applied. The proof is given in Appendix $\mathrm{C}$ and the iterative algorithm is given in Alg. 1.

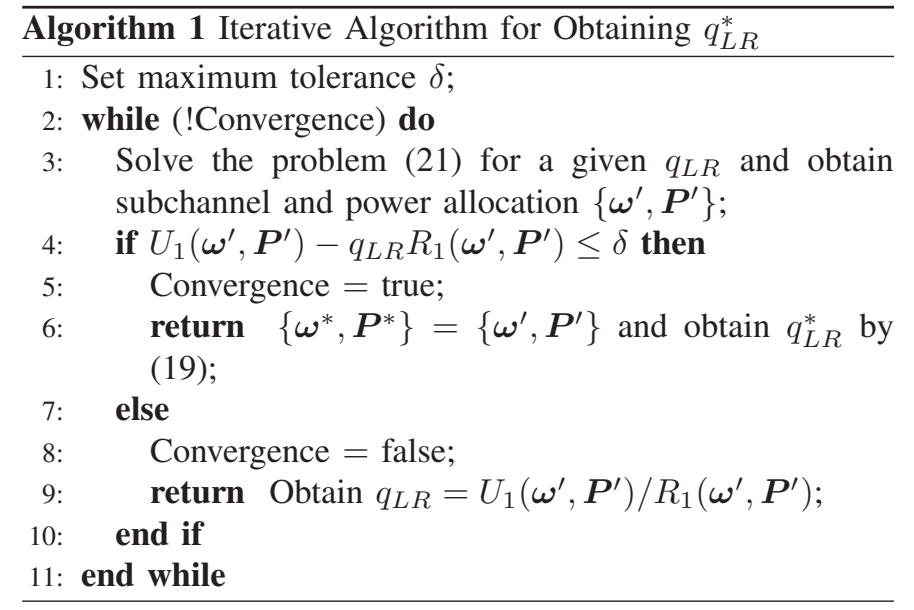




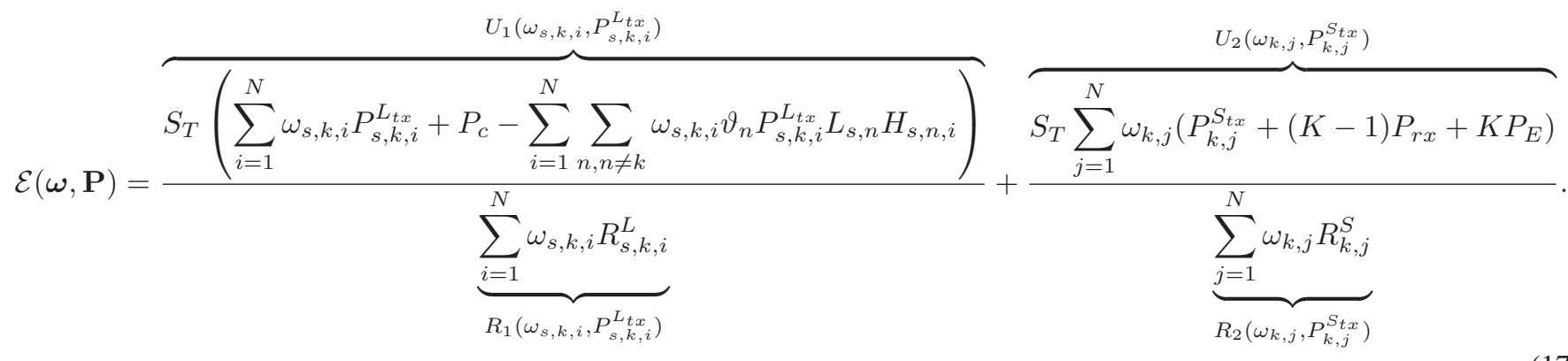

During the iteration, in order to achieve $q_{L R}^{*}$, we need to address the following problem with $q_{L R}$ :

$$
\min _{\omega_{s, k, i}, P_{s, k, i}^{L_{t x}}} U_{1}\left(\omega_{s, k, i}, P_{s, k, i}^{L_{t x}}\right)-q_{L R} R_{1}\left(\omega_{s, k, i}, P_{s, k, i}^{L_{t x}}\right),
$$

s.t.

$$
\begin{aligned}
& \sum_{k=1}^{K} \omega_{s, k, i}=1, \\
& R_{s, k, i}^{L} \geq R_{L, \min }, \\
& \sum_{i=1}^{N} \omega_{s, k, i} P_{s, k, i}^{L_{t x}} \leq P_{s, \max } .
\end{aligned}
$$

Basically, such problem is a non-convex optimization problem due to the involved integer programming. Tackling the mix convex and combinatorial optimization problem requires a prohibitively high complexity w.r.t. $K$ and $N$. Another solution which can balance the computational complexity and optimality can be obtained when addressing such problem in the dual domain. For the formulated optimization problem, as the convexity does not hold (e.g., mixed integer programming), addressing it in dual domain may result in a duality gap between primal and dual problem. As discussed and proved in [28], in the considered multi-carrier systems, the duality gap of such a non-convex resource allocation problem satisfying the time-sharing condition is negligible as the number of subcarriers becomes sufficiently large e.g., 64. To address the problem, we relax $\omega_{s, k, i}$ to be $[0,1]$ instead of a Boolean. Then, $\omega_{s, k, i}$ can be interpreted as a time sharing factor for utilizing subchannel. As one can see, the optimization problem obviously is able to satisfy the time-sharing condition, it can be solved by using the dual method and the solution is asymptotically optimal [28]. The same procedure can be used for achieving $\omega_{k, j}^{*}$ and $P_{k, j}^{S_{t x *}}$.

2) Dual Problem Formulation and Decomposition: In this part, we solve the resource allocation optimization problem of LR link by solving its dual for a given value of $q_{L R}$. Given the subchannel allocation is done, the Lagrangian function of the primal problem (21) can be given as,

$$
\begin{aligned}
& \mathcal{L}(\mathbf{P}, \mu, \theta)=U_{1}\left(P_{s, k, i}^{L_{t x}}\right)-q_{L R} R_{1}\left(P_{s, k, i}^{L_{t x}}\right)- \\
& \mu\left(R_{s, k, i}^{L}-R_{L, \min }\right)-\theta\left(P_{s, \max }-P_{s, k, i}^{L_{t x}}\right),
\end{aligned}
$$

where $\mu, \theta$ are the lagrange multipliers associated with different constraints. Therefore, the dual problem is

$$
\max _{\mu, \theta} \min _{\mathbf{P}} \mathcal{L}(\mathbf{P}, \mu, \theta) .
$$

By using Lagrange dual decomposition, the dual problem (24) can be decomposed into two layers, minimization of (23) which is the inner problem and maximization of (24) which is the outer problem. The dual problem can be solved by addressing both problems iteratively, where in each iteration, the optimal power allocation and subchannel allocation can be obtained by using the KKT conditions for a fixed set of Lagrange multipliers, and the outer problem is solved using the (sub)gradient method [29].

Using convex optimization techniques and applying the KKT conditions, the closed-form optimal power allocation on subcarrier $i$ for user $k$ for a given $q_{L R}$ can be obtained as

$$
P_{s, k, i}^{L_{t x *} *}=\left[\frac{q_{L R}+\mu}{\ln 2 \Omega_{n}}-\frac{1}{\Gamma_{n}}\right]^{+},
$$

where $\Omega_{n}=S_{T}\left(1-\sum_{n \neq k}^{K} \vartheta_{n} L_{s, n} H_{s, n, i}\right)-\mu$ and $\Gamma_{n}=$ $\frac{L_{s, n} H_{s, n, i}}{\sigma_{z}}$. Meanwhile, in order to obtain the optimal subchannel allocation $\omega_{s, k, i}^{*}$, we take the derivative of the subproblem w.r.t. $\omega_{s, k, i}$, which yields

$$
\Theta_{i}=\frac{\partial \mathcal{L}(\boldsymbol{\omega}, \mu, \theta)}{\partial \omega_{s, k, i}}=\Psi,
$$

where

$$
\begin{aligned}
& \Psi=S_{T}\left(P_{s, k, i}^{L_{t x}}+P_{r x}+P_{E}-\sum_{n \neq k} \vartheta_{k} P_{s, k, i}^{L_{t x}} L_{s, n} H_{s, n, i}\right)-\left(q_{L R}+\mu\right) \\
& +\left(1+\log _{2}\left(1+\frac{P_{s, k, i}^{L_{t x}} L_{s, k} H_{s, k, i}}{\sigma_{z}^{2}}\right)-\frac{P_{s, k, i}^{L_{t x}} L_{s, k} H_{s, k, i} / \ln 2 \sigma_{z}^{2}}{1+P_{s, k, i}^{L_{t x}} L_{s, k} H_{s, k, i} / \sigma_{z}^{2}}\right) .
\end{aligned}
$$

Thus, the subchannel allocation is given by

$$
\omega_{s, k, i}^{*}= \begin{cases}1, & \text { if } i=\arg \max _{d} \Theta_{d}, \\ 0, & \text { otherwise. }\end{cases}
$$

Moreover, the subgradient method with guaranteed convergence can be used to address the Lagrange multiplier which leads to [30]

$$
\mu^{l+1}=\mu^{l}+\epsilon_{\mu}\left(R_{L, \min }-R_{s, k, i}^{L}\right),
$$




$$
\theta^{l+1}=\mu^{l}+\epsilon_{\theta}\left(P_{s, k, i}^{L_{t x}}-P_{s, \max }\right),
$$

where $\theta^{l+1}$ and $\mu^{l+1}$ are the values of $\theta$ and $\mu$ at $l+1$ iterations. $\epsilon_{\theta}$ and $\epsilon_{\mu}$ are the corresponding step sizes. Since the problem (21) is a convex optimization problem, it is guaranteed that the iteration between the outer and the inner problems converges to the primal optimal solution of (21).

To summarize the iterative algorithm between inner and master problems, the multiplier updates can be interpreted as the pricing adjustment [31]. Particularly, if the demand of the radio resources exceeds the supply, then the gradient method will raise the prices via adjusting the Lagrange multipliers in the next iteration; otherwise, it will reduce the shadow prices until it is not out of limits. By combining the gradient updates and the subchannel allocation criterion, only one subchannel is selected eventually even though time-sharing is considered for solving the transformed problem in (21). The details of the subchannel and power allocation algorithm is presented in Alg. 2.

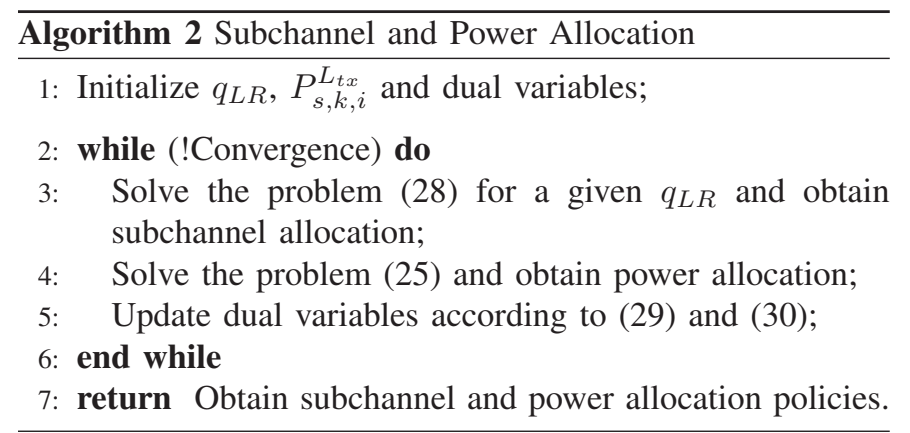

We have presented the scheme on how to address the minimization of $\mathcal{E}_{L R}\left(\omega_{s, k, i}, P_{s, k, i}^{L_{t x}}\right)$. The same procedure can be applied to obtain the optimal solution of minimizing $\mathcal{E}_{S R}\left(\omega_{k, j}, P_{k, j}^{S_{t x}}\right)$. Then we are able to obtain the solution set of (15) when considering optimal $k$ is selected.

\section{User Scheduling Scheme}

For the user scheduling problem, the goal is to select one MT to act as IMT when BS is transmitting data segment and as the data transmitter when delivering data to other MTs after receiving from BS. Therefore, with the assumption that subchannel and power allocations have been done, we are aiming to find a MT that can achieve the best energy efficiency performance considering both LR and SR links. When subchannel and power allocations are done, the objective function (15) can be reformed as,

$$
\min \mathcal{E}(\boldsymbol{\rho})=\frac{U_{1}(\boldsymbol{\rho})}{R_{1}(\boldsymbol{\rho})}+\frac{U_{2}(\boldsymbol{\rho})}{R_{2}(\boldsymbol{\rho})},
$$

where

$$
\begin{aligned}
U_{1}(\boldsymbol{\rho}) & =\sum_{k=1}^{K} \rho_{k} S_{T}\left(P_{s, k, i}^{L_{t x}}+P_{B}+P_{r x}+P_{E}\right. \\
& \left.-\sum_{n, n \neq k} \vartheta_{n} P_{s, k, i}^{L_{t x}} L_{s, n} H_{s, n, i}\right)
\end{aligned}
$$

$$
\begin{gathered}
U_{2}(\boldsymbol{\rho})=\sum_{k=1}^{K} \rho_{k} S_{T}\left(P_{k, j}^{S_{t x}}+P_{r x}+2 P_{E}\right), \\
R_{1}(\boldsymbol{\rho})=\sum_{k=1}^{K} \rho_{k} R_{s, k, i}^{L}, \\
R_{2}(\boldsymbol{\rho})=\sum_{k=1}^{K} \rho_{k} R_{k, j}^{S} .
\end{gathered}
$$

The reformed problem (31) also subjects to constraints in (16). Consequently, we can obtain the user scheduling criteria as,

$$
\rho_{k}^{*}= \begin{cases}1, & \text { if } k=\arg \min _{a} \Phi_{a} \\ 0, & \text { otherwise }\end{cases}
$$

where

$$
\Phi_{a}=\frac{U_{1}\left(\rho_{a}\right)}{R_{1}\left(\rho_{a}\right)}+\frac{U_{2}\left(\rho_{a}\right)}{R_{2}\left(\rho_{a}\right)} .
$$

\section{Solution Description}

The proposed solution involves two subproblems, which are resource (subchannel and power) allocation and user scheduling, and they are interconnected hierarchically. The convergence is guaranteed since in the sub-layer, the two transferred problems are linear for user scheduling indicator and convex for the resource allocation, respectively. The solution is illustrated in Alg. 3.

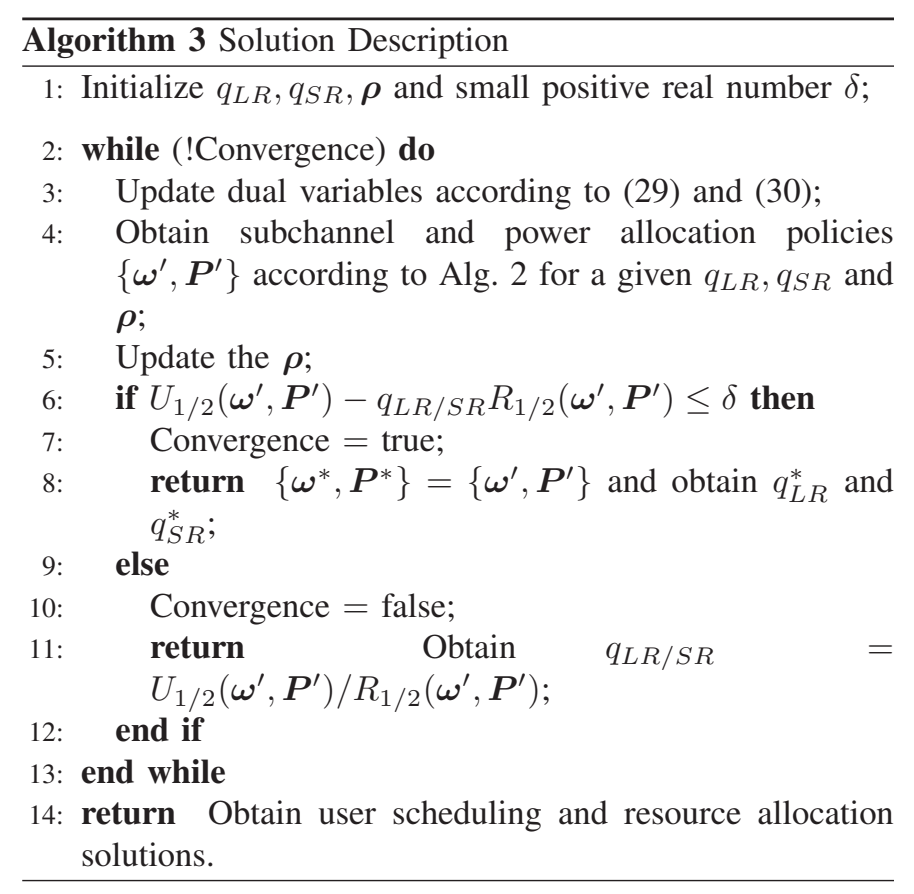




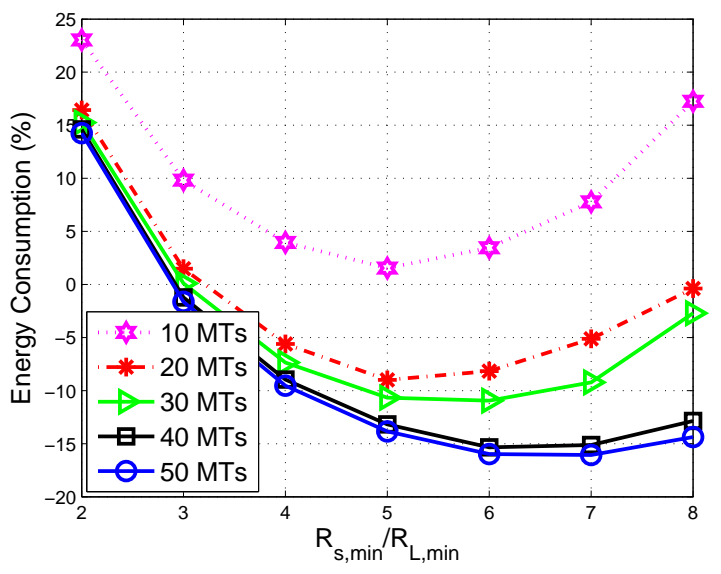

Figure 3. User energy consumption of CMC

\section{Simulation Results}

\section{A. Simulation Setting}

We present the performance evaluation in this section. For LR transmission link, the Stanford University SUI-3 channel model is used and modified to include multipath effects [32] with central frequency is $2 \mathrm{GHz}$. We use the 3-tap channel and signal fading follows Rician distribution. We choose the number of subchannels $N$ to be 64 , so the duality gap can be ignored [28]. Flat quasi-static fading channels are adopted, hence the channel coefficients are assumed to be constant during a complete data transmission, and can vary one to another independently. For the SR transmission link, the path loss follows the IEEE 802.11 ac standards with $5 \mathrm{GHz}$ central frequency. We consider the frequency bandwidths on both LR and SR are equal so no extra frequency band is needed. The noise variance is assumed 1 for simplicity. Although the baseband power $P_{E}$ and $P_{B}$ are not constant in general and their values depend on the features of circuit design, in this work it is out of the scope and we assume they are fixed according to [16]. The conversion efficiency is assumed as $\vartheta_{k}=0.5, \forall k$ for simplicity. To illustrate the energy saving performance, we compare our resource allocation scheme with pure multicast transmission, that is, the reference energy consumption is the one when BS use multicast to deliver all data to every MT as the "conventional transmission" shown in Fig. 1. In the user location setup, we consider BS is about $500 \mathrm{~m}$ from MTs and MTs are randomly located in a $50 \times 50 \mathrm{~m}^{2}$ square.

\section{B. Performance Evaluation}

In the following figures, the energy consumption performance of the proposed scheme is examined. First, the energy saving performance of CMC is examined in Fig. 3 where only energy consumption of MTs are shown. For each scheduling interval, the energy consumption ratio (EC) is obtained by normalized the presented system with conventional multicasting scheme, i.e.

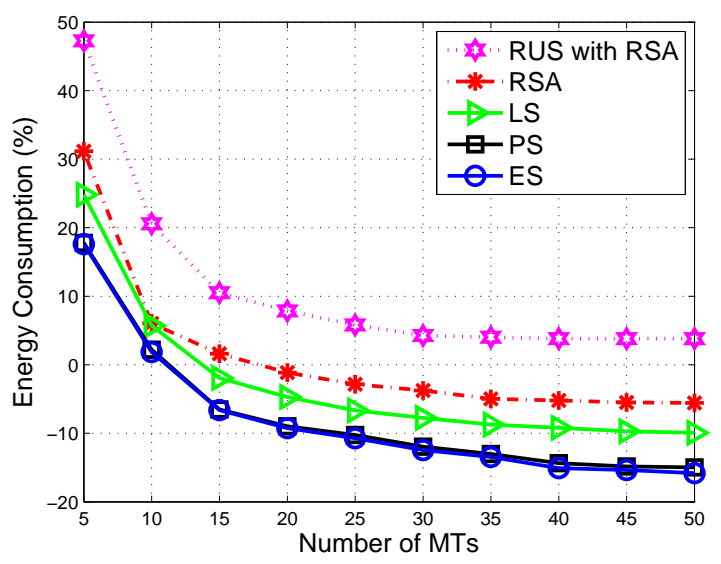

Figure 4. User energy consumption, with energy harvesting

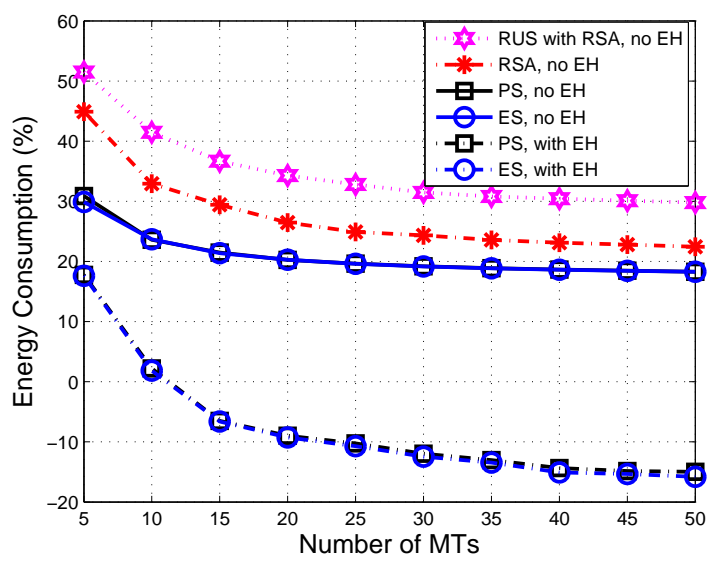

Figure 5. User energy consumption, with/without energy harvesting

$$
E C=\frac{E_{k, i, j}-\sum_{n, n \neq k}^{K} Q_{s, n, i}}{\sum_{k}^{K} E_{s, k, i}^{L_{r x}}} \times 100 \% .
$$

In Fig. 3, we fix the $R_{L, \min }=1 b p s / H z$ and vary the value of $R_{S, \min }$ to illustrate energy saving benefits of CMC and the effectiveness of advocating SR for CMC. It can be noticed that at first, when $R_{S, \text { min }}$ increases, the energy consumption of CMC is decreased. This is due to the fact that the time durations for transmitting and receiving are reduced so as the consumed energy. Since higher data rate requires higher transmit power consumption, at certain level the energy consumption is increased. For example, for the case that there are 10 MTs inside CMC, the best option for obtaining maximized energy saving is $R_{S, \min } / R_{L, \min }=5$. The negative value on the y-axis of Fig. 3 implies that the harvested energy at MTs is higher than the consumed energy, which means that the SWIPT is appreciated for the MTs who are facing energy consumption problems. Moreover, one can find that the CMC consists of more MTs can improve the energy saving potential.

In Figs. 4 and 5, we assume $R_{S, \min } / R_{L, \min }=5$ and present the energy consumption performance of all MTs in 
a CMC to show the effectiveness of proposed scheme (PS) for the CMC. We compare our proposed scheme (PS) with following reference benchmarks:

- the simulation results when random subchannel allocation (RSA) is used instead of the proposed one;

- the simulation results when the random user scheduling (RUS) together with RSA is also considered;

- the results obtained by exhaustive searching of IMT and subchannels (ES).

In order to see the wireless power transfer impact, we plot the performance of using SWIPT in Fig. 4. Moreover, to illustrate the impact of the time-sharing conditions on addressing the formulated problem, we present the performance when the number of subchannels $N$ to be 8 (LS) in Fig. 4. In addition, the comparison of the performance with SWIPT and without SWIPT is illustrated in Fig. 5. In general, we can see that the CMC is able to reduce the energy consumption in both cases. The energy saving is at least $50 \%$. Even for the cases without energy harvesting, the energy saving for the MTs can be up to $80 \%$. When the MTs are able to harvest energy from the RF signal, the energy consumption performance is improved further. We can also observe that when $N=8$, the system performance is worse than the proposed one, which also confirms that the time-sharing condition is important for addressing the formulated problem [28]. In Fig. 5, one can see that enabling wireless power transfer is able to improve the energy consumption performance up to $30 \%$, which evidences the significance of SWIPT technique. In Fig. 6, we compare our proposed scheme with the algorithms presented in [33] and [34]. We refer the one in [33] as "JC", where a user selection scheme is proposed with the joint consideration of user's energy, LR data rate, distance to other users, and mobility. The Select Best (SB) was modified from the one proposed in [34], of which the target is to select the user with the best channel condition to BS. From Fig. 6, it can be found that the proposed scheme has superior energy saving performance over the others and JC algorithm has the worst energy saving gain. This is mainly due to the proposed user selection algorithm focuses more on the energy saving criteria, and the proposed resource allocation scheme can further improve the system performance. To summarize the observations, Figs. 4 and 5 demonstrate that

- the use of CMC is able to reach a promising energy saving gain when comparing with traditional multicasting transmission.

- the PS has supervisor performance over RSA, which induces that carefully designing the resource allocation scheme for CMC is necessary.

- by recycling energy from RF signal, the energy consumption performance can be improved.

- the performance of our proposed scheme is very close to the optimal one.

In Fig. 7, the energy consumption of the overall system including both BS and MTs is presented. We compare the system performance of PS with the one of RSA and the one of RUS and RSA. The energy consumption ratio is obtained

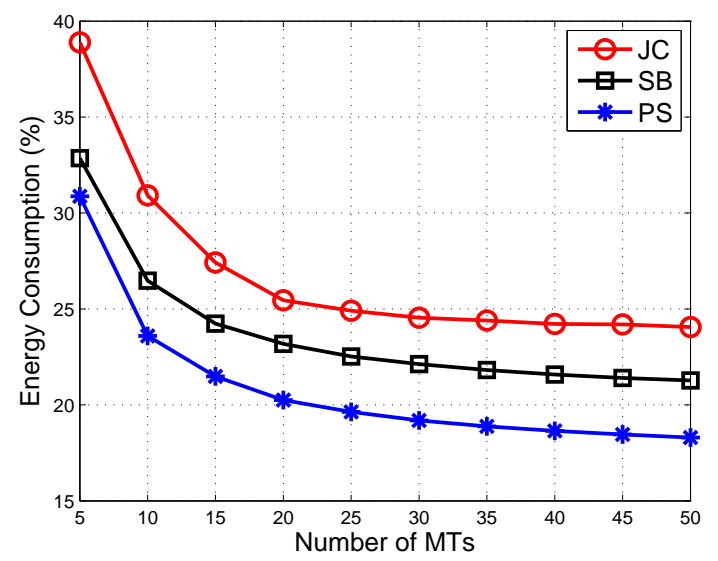

Figure 6. Comparison of user energy consumption performance

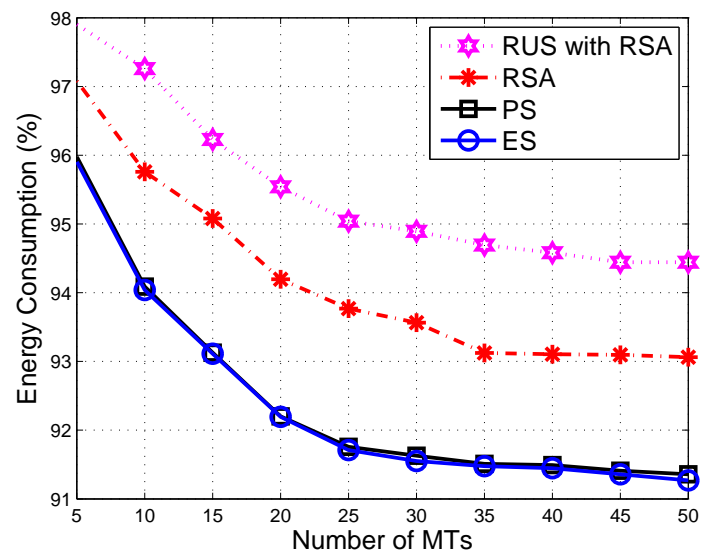

Figure 7. System energy consumption, with both BS and user energy consumption

by the energy consumption of proposed scheme normalized by the one when multicasting is invoked, i.e.,

$$
E C=\frac{E_{s}^{L_{t x}}+E_{k, i, j}-\sum_{n, n \neq k}^{K} Q_{s, n, i}}{\sum_{k}^{K} E_{s, k, i}^{L_{r x}}+E_{s, t r a}^{L_{t x}}} \times 100 \% .
$$

where $E_{s, t r a}^{L_{t x}}$ is the traditional multicasting transmit energy consumption for single data segment. When jointly considering energy consumption of BS and MTs, the energy saving is up to $9 \%$ compared to multicasting transmission. This is mainly due to the fact that energy saving of BS is fairly lower than the one of MTs and energy consumption of BS dominates in the overall system energy consumption. Thus, the proposed scheme has less impact on the energy consumption performance of BS.

\section{CONCLUSION}

In this paper we have investigated the problem of resource allocation and user scheduling for OFDMA networks with collaborative mobile clouds. By assuming that the mobile cloud containing numbers of hybrid information decoding and energy harvesting user equipments, we proposed an algorithm 
which can noticeably obtain energy efficiency performance. The joint optimization problem was solved by addressing two sub-problems including opportunistic selection of information decoding receiver, subchannel and power allocations with the objective of minimizing the energy consumption. Simulation results illustrated the performance gains of the proposed resource allocation and user scheduling schemes compared to the other baseline schemes, and unveiled the potential gain of using wireless power transfer for the MTs. The presented results demonstrate that 1 ) The collaborative mobile clouds system is able to reduce the energy consumption of system, especially at MTs side during the receiving process; 2) the proposed resource allocation and user scheduling algorithms is able to reduce the total transmit energy consumption and improve the energy efficiency performance; 3) the wireless power transfer is able to further improve the energy saving performance of MTs. Therefore, properly invoking the wireless power transfer is able to provide a promising solution for the future energy consumption problems.

As one future direction, the work could be extended to the cloud formulation problem where resource allocation and multiple CMC formulation can be jointly studied. Another extension of this work is to investigate the trade-off between energy efficiency and user fairness in the future since user fairness is also an important factor in the user cooperation schemes. Social and user fairness factors will be considered when allocating the radio resource and scheduling user for data assignment so that fairness among the CMC is able to be guaranteed.

\section{APPENDIX A}

\section{Proof of Theorem 1}

To start with, recalling the energy consumption model

To facilitate the the following analysis, we assume that the subchannels $i$ and $j$ are allocated to scheduled MT $k$ optimally for the transmission process, i.e., $\rho_{k}=\omega_{s, k, i}=\omega_{k, j}=1$. Substitute (40) into (11) we can arrive

$$
\left\{\begin{aligned}
E_{s}^{L_{t x}} & =\frac{\left(P_{s, k, i}^{L_{t x}}+P_{B}\right) S_{T}}{R_{s, k, i}^{L}} \\
E_{k, i, j} & =\frac{\left(P_{r x}+P_{E}\right) S_{T}}{R_{s, k, i}^{L}}+\frac{\left(P_{k, j}^{S_{t x}}+P_{E}\right) S_{T}}{R_{k, j}^{S}}+\frac{\left(P_{r x}+P_{E}\right) S_{T}}{R_{k, j}^{S}} \\
Q_{s, n, i} & =\frac{\vartheta_{n} P_{s, k, i}^{L} L_{s, n} H_{s, n, i} S_{T}}{R_{s, k, i}^{L}}
\end{aligned}\right.
$$

we use $S_{T}=1$. Note that the power allocation policy $\mathbf{P}$ is $\mathbf{P}=\left\{P_{s, k, i}^{L_{t x}}, P_{k, j}^{S_{t x}}\right\}$. We can see $R_{s, k, i}^{L}$ and $R_{k, j}^{S}$ are the concave functions w.r.t to the power allocation $P_{s, k, i}^{L_{t x}}$ and $P_{k, j}^{S_{t x}}$, respectively. Thus, the objective function is strictly quasi-convex function w.r.t. $P_{s, k, i}^{L_{t x}}$ and $P_{k, j}^{S_{t x}}$, respectively [26]. Further we can prove that the objective function is first monotonically non-increase and then monotonically non-decrease. The proof can be easily obtained by $\left.\frac{\partial \mathcal{E}(\mathbf{P})}{\partial \mathbf{P}}\right|_{\mathbf{P} \rightarrow 0} \leq 0$ and $\left.\frac{\partial \mathcal{E}(\mathbf{P})}{\partial \mathbf{P}}\right|_{\mathbf{P} \rightarrow \infty}>0$.

\section{APPENDIX B}

\section{Proof of Theorem 2}

Similar to the previous proof, we assume that that the subchannels $i$ and $j$ are allocated to scheduled MT $k$ optimally for the transmission process, i.e., $\rho_{k}=\omega_{s, k, i}=\omega_{k, j}=1$. Suppose $\mathcal{P}$ is the solution set and let $P_{0}$ be a solution of (19), then we have

$$
q_{0}=\frac{U_{1}\left(P_{0}\right)}{R_{1}\left(P_{0}\right)} \leq \frac{U_{1}(P)}{R_{1}(P)}, \forall P \in \mathcal{P} .
$$

Consequently, we can arrive

$$
U_{1}(P)-q_{0} R_{1}(P) \geq 0, \forall P \in \mathcal{P},
$$

and

$$
U_{1}\left(P_{0}\right)-q_{0} R_{1}\left(P_{0}\right)=0, \forall P \in \mathcal{P} .
$$

From (43) we see that $\min \left\{U_{1}(P)-q_{0} R_{1}(P) \mid P \in \mathcal{P}\right\}=0$. From (44) we observe that the minimum value is taken when $P=P_{0}$. Therefore, the necessary condition can be proved.

To prove the sufficient condition, let $P_{0}$ be a solution of (20), then we have

$$
U_{1}(P)-q_{0} R_{1}(P) \geq U_{1}\left(P_{0}\right)-q_{0} R_{1}^{L}\left(P_{0}\right)=0, \forall P \in \mathcal{P} .
$$

Hence

$$
U_{1}(P)-q_{0} R_{1}^{L}(P) \geq 0, \forall P \in \mathcal{P},
$$

and

$$
U_{1}\left(P_{0}\right)-q_{0} R_{1}^{L}\left(P_{0}\right)=0, \forall P \in \mathcal{P} .
$$

From (46) we have $\frac{U_{1}(P)}{R_{1}^{L}(P)} \geq q_{0}$, that $q_{0}$ is the minimum of (19). From (47) we have $\frac{U_{1}\left(P_{0}\right)}{R_{1}^{L}\left(P_{0}\right)}=q_{0}$, that is $P_{0}$ is a solution of (19)

$$
\mathcal{E}(\mathbf{P})=\frac{\left(P_{s, k, i}^{L_{t x}}+P_{B}\right) S_{T}}{R_{s, k, i}^{L}}+\frac{\left(P_{r x}+P_{E}\right) S_{T}}{R_{s, k, i}^{L}}+\frac{\left(P_{k, j}^{S_{t x}}+P_{E}\right) S_{T}}{R_{k, j}^{S}}
$$$$
+\frac{\left(P_{r x}+P_{E}\right) S_{T}}{R_{k, j}^{S}}-\frac{\sum_{n, n \neq k} \vartheta_{n} P_{s, k, i}^{L_{t x}} L_{s, n} H_{s, n, i} S_{T}}{R_{s, k, i}^{L}}
$$$$
=\frac{P_{s, k, i}^{L_{t x}}-\sum_{n, n \neq k} \vartheta_{n} P_{s, k, i}^{L_{t x}} L_{s, n} H_{s, n, i}+C_{1}}{R_{s, k, i}^{L}}+\frac{\left(P_{k, j}^{S_{t x}}+C_{2}\right)}{R_{k, j}^{S}} \text {, }
$$

$$
f\left(q^{\prime}\right)=\min _{\boldsymbol{\omega}, \boldsymbol{P}} U_{1}(\boldsymbol{\omega}, \boldsymbol{P})-q^{\prime} R_{1}(\boldsymbol{\omega}, \boldsymbol{P})
$$

where $\boldsymbol{\omega}=\left\{\omega_{s, k, i}\right\}$ and $\boldsymbol{P}=\left\{P_{s, k, i}^{L_{t x}}\right\}$. Then assuming $q^{\prime}>$ $C_{1}=P_{B}+P_{r x}+P_{E}$ and $C_{2}=P_{r x}+2 P_{E}$ are constant for the considered model [16]. For the sake of simplicity $q^{\prime}$ and considering two optimal resource allocation policies, 
$\left\{\boldsymbol{\omega}^{\prime}, \boldsymbol{P}^{\prime}\right\}$ and $\left\{\boldsymbol{\omega}^{\prime}, \boldsymbol{P}^{\prime}\right\}$ for $f\left(q^{\prime}\right)$ and $f\left(q^{\prime}\right)$ respectively, we have

$$
\begin{aligned}
f\left(q^{\prime}\right) & =\min _{\boldsymbol{\omega}, \boldsymbol{P}} U_{1}(\boldsymbol{\omega}, \boldsymbol{P})-q^{\prime} R_{1}(\boldsymbol{\omega}, \boldsymbol{P}) \\
& =U_{1}\left(\boldsymbol{\omega}^{\prime}, \boldsymbol{P}^{\prime}\right)-q^{\prime} R_{1}\left(\boldsymbol{\omega}^{\prime}, \boldsymbol{P}^{\prime}\right) \\
& <U_{1}\left(\boldsymbol{\omega}^{\prime}, \boldsymbol{P}^{\prime}\right)-q^{\prime} R_{1}\left(\boldsymbol{\omega}^{\prime}, \boldsymbol{P}^{\prime}\right) \\
& \leq U_{1}\left(\boldsymbol{\omega}^{\prime}, \boldsymbol{P}^{\prime}\right)-q^{\prime} R_{1}\left(\boldsymbol{\omega}^{\prime}, \boldsymbol{P}^{\prime}\right) \\
& =f\left(q^{\prime}\right) .
\end{aligned}
$$

Therefore, we can see $f(q)$ is a strong monotonic decreasing function, i.e. $f\left(q^{\prime}\right)<f\left(q^{\prime}\right)$, if $q^{\prime}>q^{\prime}$. Suppose $\left\{\boldsymbol{\omega}^{l}, \boldsymbol{P}^{l}\right\}$ is the optimal resource allocation policies in the $l$ th iteration and $q^{l} \neq q^{*}$ and $q^{l+1} \neq q^{*}$. We can observe that $q^{l}>0$ and $q^{l+1}>0$. Since in Algorithm 1, we obtain $q^{l+1}=U_{1}\left(\boldsymbol{\omega}^{l}, \boldsymbol{P}^{l}\right) / R_{1}\left(\boldsymbol{\omega}^{l}, \boldsymbol{P}^{l}\right)$. Thus one can arrive

$$
\begin{aligned}
f\left(q^{l}\right) & =U_{1}\left(\boldsymbol{\omega}^{l}, \boldsymbol{P}^{l}\right)-q^{l} R_{1}\left(\boldsymbol{\omega}^{l}, \boldsymbol{P}^{l}\right) \\
& =R_{1}\left(\boldsymbol{\omega}^{l}, \boldsymbol{P}^{n}\right)\left(q^{l+1}-q^{l}\right) .
\end{aligned}
$$

As $f\left(q^{l}\right)>0$ and $R_{1}\left(\boldsymbol{\omega}^{l}, \boldsymbol{P}^{l}\right)$, we have $q^{l+1}>q^{l}$. Therefore, we can see that as long as the number of iterations is large enough, $F\left(q^{l}\right) \rightarrow 0$ and $F\left(q^{l}\right)$ satisfies the optimality condition as presented in Theorem $\mathbf{2}$. To this end, the convergence of Algorithm 1 can be proved.

\section{REFERENCES}

[1] J. Yang, and S. Ulukus, "Optimal Packet Scheduling in an Energy Harvesting Communication System," IEEE Trans. on Commun., vol. 60, no. 1, pp. 220-230, Jan. 2012.

[2] C. K. Ho, and R. Zhang, "Optimal Energy Allocation for Wireless Communications With Energy Harvesting Constraints," IEEE Trans. Signal Proc., vol. 60, no. 9, pp. 4808-4818, Sep. 2012.

[3] J. Gong, S. Zhou, and Z. Niu, "Optimal Power Allocation for Energy Harvesting and Power Grid Coexisting Wireless Communication Systems," IEEE Trans. on Commun., vol. 61, no. 7, pp. 3040-3049, July 2013.

[4] L. R. Varshney, "Transporting Information and Energy simultaneously," in Proc. of IEEE ISIT'08, Toronto, Canada, July 2008.

[5] P. Grover, and A. Sahai, "Shannon Meets Tesla: Wireless Information and Power Transfer," in Proc. of IEEE ISIT'10, Austin, TX, June 2010.

[6] K. Huang, and E. Larsson, "Simultaneous Information and Power Transfer for Broadband Wireless Systems," IEEE Trans. Signal Proc., vol. 61, no. 23, pp. 5972-5986, Dec. 2013.

[7] R. Zhang, and C. K. Ho, "MIMO Broadcasting for Simultaneous Wireless Information and Power Transfer," IEEE Trans. Wireless Commun., vol. 12, no. 5, pp. 1989-2001, May 2013.

[8] X. Zhou, R. Zhang, and C-K Ho, "Wireless Information and Power Transfer: Architecture Design and Rate-Energy Tradeoff," in Proc. of GLOBECOM'12, Anaheim, CA, Dec. 2012.

[9] L. Liu, R. Zhang, and K-C Chua, "Wireless Information Transfer with Opportunistic Energy Harvesting," IEEE Trans. Wireless Commun., Vol. 12, No. 1, pp. 288-300, Jan. 2013.

[10] Z. Ding, and H. V. Poor, "Cooperative Energy Harvesting Networks with Spatially Random Users," IEEE Signal Processing Letters, vol. 20, no. 12, pp. 1211-1214, Dec. 2013.

[11] D. W. K. Ng, E. Lo, and R. Schober, "Energy-Efficient Resource Allocation in Multiuser OFDM Systems with Wireless Information and Power Transfer," in Proc. of IEEE WCNC'13, Shanghai, China, Apr. 2013.

[12] D. W. K. Ng, E. Lo, and R. Schober, "Wireless Information and Power Transfer: Energy Efficiency Optimization in OFDMA Systems," IEEE Trans. Wireless Commun., vol. 12, no. 12, pp. 6352-6370, Dec. 2013.

[13] X. Chen, X. Wang, and X. Chen, "Energy-Efficient Optimization for Wireless Information and Power Transfer in Large-Scale MIMO Systems Employing Energy Beamforming", IEEE Wireless Commun. Letters, vol. 2, no. 6, pp. 667-670, Dec. 2013.
[14] M. V. Pederson, and F. H. P. Fitzek, "Mobile Clouds: The New Content Distribution Platform," Proceeding of IEEE, vol. 100, pp. 1400-1403, 2012.

[15] L. Al-Kanj, Z. Dawy, and E. Yaacoub, "Energy-Aware Cooperative Content Distribution over Wireless Networks: Design Alternatives and Implementation Aspects," IEEE Communications Surveys and Tutorials, vol. 15, no. 4, pp. 1736-1760, Dec. 2013.

[16] Z. Chang and T. Ristaniemi, "Energy Efficiency of Collaborative OFDMA Mobile Cluster," in Proc. of IEEE CCNC'13, Las Vegas, NV, Jan. 2013.

[17] Z. Yin, F. R. Yu, S. Bu, and Z. Han, "Joint Cloud and Wireless Networks Operations in the Mobile Cloud Computing Environment with Telecom Operator Cloud," IEEE Trans. Wireless Commun., vol. 14, no. 7, pp. 4020-4033, July 2015.

[18] D. Niyato, P. Wang, E. Hossain, W. Saad, and Z. Han, "Game Theoretic Modeling of Cooperation among Service Providers in Mobile Cloud Computing Environments," in proc. of IEEE Wireless Communications and Networking Conference, Paris, France, April 2012.

[19] L. Al-Kanj, Z. Dawy, W. Saad, and E. Kutanoglu, "Energy-Aware Cooperative Content Distribution Over Wireless Networks: Optimized and Distributed Approaches," IEEE Trans. Veh. Technol, vol. 62, no. 8, pp. 3828-3847, Aug. 2013.

[20] E. Yaacoub, Z. Dawy, S. Sharafeddine, and A. Abu-Dayya, "Joint Energy-Distortion Aware Algorithms for Cooperative Video Streaming over LTE Networks", EURASIP/Elsevier Signal Processing: Image Communication, 28(9), pp. 1114-1131, 2013.

[21] W. Saad, Z. Han, A. Hjorungnes, D. Niyato, and E. Hossain, " Coalition Formation Games for Distributed Cooperation Among Roadside Units in Vehicular Networks," IEEE Selected Areas in Communications, vol. 29, no. 1, pp.48-60, Jan. 2011

[22] T. Wang, L. Song, Z. Han and B. Jiao, "Dynamic Popular Content Distribution in Vehicular Networks using Coalition Formation Games," IEEE Selected Areas in Communications, vol. 31, no. 9, pp. 538-547, Sep. 2013.

[23] Z. Chang, T. Ristaniemi, and Z. Niu, "Energy Efficient Grouping and Scheduling For Content Sharing based Collaborative Mobile Cloud," in Proc. of ICC'14, Sydney, Australia, June 2014.

[24] Z. Chang, J. Gong and T. Ristaniemi, "Energy Efficient Resource Allocation for Collaborative Mobile Cloud with Hybrid Receiver," in Proc. of IEEE INFOCOM'14 workshop, Toronto, Canada, April 2014

[25] Z. Chang, and T. Ristaniemi, "Efficient Use of Multicast and Unicast in Collaborative OFDMA Mobile Cluster," in Proc. of VTC'13-spring, Dresden, Germany, Jun. 2013.

[26] S. Boyd, and L. Vandenberghe, Convex Optimization, Cambridge UK: Cambridge University Press, 2004.

[27] W. Dinkelbach, "On Nonlinear Fractional Programming," Management Science, vol. 13, pp. 492-498, Mar. 1967.

[28] W. Yu, and R. Lui, "Dual Methods for Nonconvex Spectrum Optimization in Multicarrier System," IEEE Trans. on Commun., vol. 54, no. 7, pp. 1310-1322, Jul. 2006.

[29] S. Boyd, and A. Mutapcic, Lecture Notes for EE364b, Standford University, 2006-2007.

[30] Z. Chang, T. Ristaniemi, and Z. Niu, "Radio Resource Allocation for Collaborative OFDMA Relay Networks with Imperfect Channel State Information," IEEE Trans. Wireless Commun., vol.13, no.5, pp. 28242835, May 2014.

[31] D. W. K. Ng, E. Lo, and R. Schober, "Energy-Efficient Resource Allocation in OFDMA Systems with Large Numbers of Base Station Antennas," IEEE Trans. Wireless Commun., vol. 11, no. 9, pp. 32923304, Sep. 2012.

[32] R. Jain, Channel Models A Tutorial1 v1.0, Feb. 2007.

[33] H. Bagheri, M. J. Salehi, B. H. Khalaj, and M. Katz, "An energy-efficient leader selection algorithm for cooperative mobile clouds," IEEE/IFIP Wireless Days, Valencia, Spain, Nov. 2013.

[34] B. Barua, Z. Khan, Z. Han, M. Latva-aho, and M. Katz, "On the Selection of Best Devices for Cooperative Wireless Content Delivery," in proc. of IEEE GLOBECOM'14, Austin, TX, Dec. 2014. 


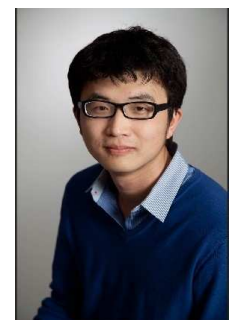

Zheng Chang (M'13) received the B.Eng. degree from Jilin University, Changchun, China in 2007 , M.Sc. (Tech.) degree from Helsinki University of Technology (Now Aalto University), Espoo, Finland in 2009 and Ph.D degree from the University of Jyväskylä, Jyväskylä, Finland in 2013. Since 2008, he has held various research positions at Helsinki University of Technology, University of Jyväskylä and Magister Solutions Ltd in Finland. During June to August in 2013, he was also a visiting researcher at Tsinghua University, China and University of Houston, TX. He has been awarded by the Ulla Tuominen Foundation, the Nokia Foundation and the Riitta and Jorma J. Takanen Foundation for his research work. Currently he is working with University of Jyväskylä and his research interests include signal processing, radio resource allocation, crosslayer optimizations for wireless networks, and green communications.

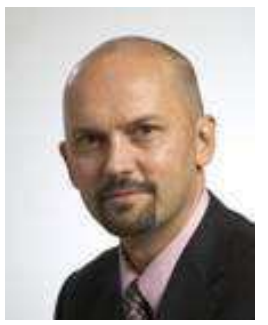

Tapani Ristaniemi (SM'11) received his M.Sc in 1995 (Mathematics), Ph.Lic. in 1997 (Applied Mathematics) and Ph.D. in 2000 (Wireless Communications), all from the University of Jyväskylä, Jyväskylä, Finland. In 2001 he was appointed as Professor in the Department of Mathematical Information Technology, University of Jyväskylä. In 2004 he moved to the Department of Communications Engineering, Tampere University of Technology, Tampere, Finland, where he was appointed as Professor in Wireless Communications. In 2006 he moved back to University of Jyväskylä to take up his appointment as Professor in Computer Science. He is an Adjunct Professor of Tampere University of Technology. In 2013 he was a Visiting Professor in the School of Electrical and Electronic Engineering, Nanyang Technological University, Singapore.

He has authored or co-authored over 150 publications in journals, conference proceedings and invited sessions. He served as a Guest Editor of IEEE Wireless Communications in 2011 and currently he is an Editorial Board Member of Wireless Networks and International Journal of Communication Systems. His research interests are in the areas of brain and communication signal processing and wireless communication systems research.

Besides academic activities, Professor Ristaniemi is also active in the industry. In 2005 he co-founded a start-up Magister Solutions Ltd in Finland, specialized in wireless system R\& D for telecom and space industries in Europe. Currently he serves as a consultant and a Member of the Board of Directors.

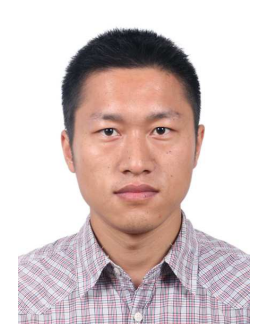

Jie Gong (S'09, M'13) received his B.S. and Ph.D degrees in Department of Electronic Engineering in Tsinghua University, Beijing, China, in 2008 and 2013, respectively. From July 2012 to January 2013, he visited Institute of Digital Communications, University of Edinburgh, Edinburgh, UK. During 2013-2015, he worked as a postdoctorial scholar in Department of Electronic Engineering in Tsinghua University, Beijing, China. He is currently an associate research fellow in School of Data and Computer Science, Sun Yat-sen University, Guangzhou, Guangdong Province, China. He was a co-recipient of the Best Paper Award from IEEE Communications Society Asia-Pacific Board in 2013. His research interests include Cloud RAN, energy harvesting and green wireless communications.

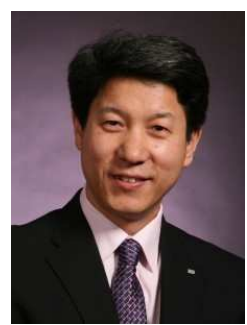

Zhisheng Niu (M'98-SM'99-F'12) graduated from Beijing Jiaotong University, China, in 1985, and got his M.E. and D.E. degrees from Toyohashi University of Technology, Japan, in 1989 and 1992 respectively. During 1992-94, he worked for Fujitsu Laboratories Ltd., Japan, and in 1994 joined with Tsinghua University, Beijing, China, where he is now a professor at the Department of Electronic Engineering. He is also a guest chair professor of Shandong University, China. His major research interests include queueing theory, traffic engineering, mobile Internet, radio resource management of wireless networks, and green communication and networks.

Dr. Niu has been an active volunteer for various academic societies, including Director for Conference Publications (2010-11) and Director for Asia-Pacific Board (2008-09) of IEEE Communication Society, Membership Development Coordinator (2009-10) of IEEE Region 10, Councilor of IEICEJapan (2009-11), and council member of Chinese Institute of Electronics (2006-11). He is now a distinguished lecturer (2012-15) and Chair of Emerging Technology Committee (2014-15) of IEEE Communication Society, a distinguished lecturer (2014-16) of IEEE Vehicular Technologies Society, a member of the Fellow Nomination Committee of IEICE Communication Society (2013-14), standing committee member of Chinese Institute of Communications (CIC, 2012-16), and associate editor-in-chief of IEEE/CIC joint publication China Communications.

Dr. Niu received the Outstanding Young Researcher Award from Natural Science Foundation of China in 2009 and the Best Paper Award from IEEE Communication Society Asia-Pacific Board in 2013. He also co-received the Best Paper Awards from the 13th, 15th and 19th Asia-Pacific Conference on Communication (APCC) in 2007, 2009, and 2013, respectively, International Conference on Wireless Communications and Signal Processing (WCSP'13), and the Best Student Paper Award from the 25th International Teletraffic Congress (ITC25). He is now the Chief Scientist of the National Basic Research Program (so called "973 Project") of China on "Fundamental Research on the Energy and Resource Optimized Hyper-Cellular Mobile Communication System" (2012-2016), which is the first national project on green communications in China. He is a fellow of both IEEE and IEICE. 